\title{
Atorvastatin attenuates plaque vulnerability by downregulation of EMMPRIN expression via COX-2/PGE2 pathway
}

\author{
XING LIANG $^{1}$, LI-XIA YANG ${ }^{1}$, RUIWEI GUO ${ }^{1}$, YANKUN SHI ${ }^{1}$, XIANHUA HOU $^{2}$, \\ ZHIHUA YANG ${ }^{1}$, XIAOBIN ZHOU ${ }^{1}$ and HONG LIU ${ }^{1}$ \\ ${ }^{1}$ Department of Cardiology, Kunming General Hospital of Chengdu Military Area, Kunming, Yunnan 650032; \\ ${ }^{2}$ Department of Neurology, Southwestern Hospital, Third Military Medical University, Chongqing 400038, P.R. China
}

Received July 14, 2015; Accepted September 6, 2016

DOI: $10.3892 / \mathrm{etm} .2017 .4062$

\begin{abstract}
Extracellular matrix metalloproteinase inducer (EMMPRIN) reportedly has a key regulatory role in matrix metalloproteinase (MMP) activities and the progression of atherosclerosis. Statins, which are anti-atherosclerotic pharmacological agents, are widely applied in clinical settings. The aim of the present study was to investigate the pharmaceutical effect of atorvastatin on EMMPRIN expression in atherosclerotic plaques. An atherosclerotic mouse model was established using apoliprotein E-deficient $\left(\mathrm{ApoE}^{-/-}\right)$mice raised on a high-fat diet. Additionally, a low ( $5 \mathrm{mg} / \mathrm{kg} /$ day $)$ or high dosage $(10 \mathrm{mg} / \mathrm{kg} /$ day $)$ of atorvastatin suspension was administered orally for eight weeks, beginning on week 7 or 11 respectively. The effects of atorvastatin on atherosclerotic plaque formation and EMMPRIN expression were subsequently determined. The THP-1 cell line was used to investigate the effect of atorvastatin on EMMPRIN expression in vitro. The results demonstrated that the high-fat diet led to vulnerable plaques (VPs) and increased EMMPRIN expression in VPs in ApoE ${ }^{-/-}$mice. Atorvastatin treatment decreased EMMPRIN expression in the aortas and plaques of $\mathrm{ApoE}^{-/}$mice. In vitro, oxidized low-density lipoprotein (ox-LDL) induced the expression of cyclooxygenase-2 (COX-2) and EMMPRIN in THP-1 macrophages, and atorvastatin inhibited ox-LDL-induced expression of PGE2, EMMPRIN and COX-2 in THP-1 macrophages. Therefore, the present data indicated that atorvastatin treatment reduces the vulnerability of atherosclerotic plaques and expression of EMMPRIN, and that the inhibitory effect of atorvastatin on EMMPRIN may occur via the COX-2/PGE2 signaling pathway in macrophages.
\end{abstract}

Correspondence to: Professor Li-Xia Yang, Department of Cardiology, Kunming General Hospital of Chengdu Military Area, 212 DaGuan Road, Kunming, Yunnan 650032, P.R. China

E-mail: doctoryanglx@163.com

Key words: extracellular matrix metalloproteinase inducer, apolipoprotein E knockout mice, atorvastatin, atherosclerosis, vulnerable plaque

\section{Introduction}

Coronary heart disease (CHD) is the most common cause of mortality worldwide (1). Acute coronary syndrome (ACS) is a severe phase of $\mathrm{CHD}$ and a predominant cause of cardiac events (2). The principal pathophysiology of ACS is the rupture of coronary plaques with subsequent thrombosis (3). The pathological characteristics of vulnerable plaques (VPs) include the presence of a soft, lipid-rich core that is covered by a thin cap of fibrous tissue and is infiltrated by a large number of inflammatory cells (4). Plaque rupture occurs in various pathological states and is typically associated with matrix metalloproteinases (MMPs). MMPs are able to degrade collagen and other extracellular matrix (ECM) components that compose the major structure of plaques and previous studies have indicated an association between the level of MMPs and plaque stability (5-7). Co-expression of extracellular matrix metalloproteinase inducer (EMMPRIN) and MMPs has been observed in macrophages in vitro and in human atheroma, particularly in the shoulder region of VP atheroma (8). The present study suggested that EMMPRIN serves a key role in regulating MMP activity in cardiovascular diseases.

Atorvastatin is an inhibitor of 3-hydroxy-3-methyl-glutaryl coenzyme A (HMG-CoA) reductase. Large randomized clinical trials have determined that statins are able to reduce the incidence of life-threatening vascular events in patients with ACS (9-11). Over the last decade, statins have become a standard treatment regimen for patients with atherosclerosis. The anti-atherosclerotic effects and concomitant lipid-lowering effects of atorvastatin have been demonstrated in previous studies. Statins have many beneficial effects, including lowering cholesterol $(12,13)$, improving endothelial function $(14,15)$ and reducing systemic inflammatory markers $(16,17)$.

As a factor in atherosclerosis, EMMPRIN mediates plaque destabilization in myocardial infarction, whereas atorvastatin has a protective role in the development of VP. However, the mechanism between EMMPRIN and atorvastatin in aortic atherosclerotic plaques is not yet understood. Therefore, in the present study, the effect of atorvastatin on EMMPRIN expression in aortic atherosclerotic plaques was investigated. 


\section{Materials and methods}

Reagents and equipment. A goat anti-EMMPRIN polyclonal antibody (sc-9757), a rabbit polyclonal antibody (sc-7951) against cyclooxygenase-2 (COX-2), dithiothreitol, aprotinin, leupeptin,phenylmethylsulfonyl fluoride(PMSF), Tween 20 and Nonidet P-40 were purchased from Santa Cruz Biotechnology, Inc., (Dallas, TX, USA). The rabbit anti-glyceraldehyde 3-phosphate dehydrogenase (GAPDH) monoclonal antibody (NB100-56875) was purchased from Novus Biologicals, LLC (Littleton, CO, USA). The goat anti-rabbit (IRDye680LT) and donkey anti-goat (IRDye800CW) secondary antibodies were purchased from LI-COR Biosciences (Lincoln, NE, USA). The RNAiso Plus, SYBR Premix Ex Taq II and the PrimeScript RT Reagent kit with gDNA Eraser were purchased from Takara Bio, Inc., (Otsu, Japan). The goat anti-rabbit (ZB-5301) and rabbit anti-goat (ZB-2306) horseradish peroxidase (HRP)-conjugated secondary antibodies, a diaminobenzidine (DAB) color reagent kit (ZLI-9017) and an ELISA kit (EIA-1112) used for the determination of prostaglandin E2 (PGE2) levels were purchased from OriGene Technologies, Inc., (Beijing, China). Atorvastatin and ezetimibe, which is a selective inhibitor of cholesterol absorption, were gifts from Pfizer, Inc. (New York, NY, USA) and Merck \& Co. (Kenilworth, NJ, USA), respectively. Polymerase chain reaction (PCR) amplification was performed using a thermal cycler PTC-200 (Bio-Rad Laboratories, Inc., Hercules, CA, USA), a nucleic acid protein analyzer DU800 (Beckman Coulter, Inc., Brea, CA, USA), a microplate reader FO39300 (Bio-Rad Laboratories, Inc.), a ChemiImager 5500 gel imaging analysis system (ProteinSimple, San Jose, CA, USA) and a Leica DMIRB inverted fluorescence microscope (Leica Microsystems, Inc., Buffalo Grove, IL, USA). RPMI 1640 medium and fetal bovine serum (FBS) were purchased from Gibco, Thermo Fisher Scientific, Inc. (Waltham, MA, USA). Phorbol 12-myristate 13-acetate (PMA), oxidized low-density lipoprotein (ox-LDL), NS-398 (an inhibitor of COX-2), PGE2 and sodium pentobarbitone (1507002) were obtained from Sigma-Aldrich (Merck Millipore, Darmstadt, Germany).

Mice and husbandry. Apolipoprotein E knockout $\left(\mathrm{ApoE}^{-/-}\right)$ male mice ( $\mathrm{n}=72$; age, 8 weeks; weight, $24 \pm 1 \mathrm{~g}$ ) were purchased from the Animal Centre of the Medical Department of Beijing University (Beijing, China). Mice were maintained under standard conditions of humidity (50-60\%) and temperature $\left(18-22^{\circ} \mathrm{C}\right)$, and were subjected to a $12 \mathrm{~h}$ hour light-dark cycle with ad libitum access to feed and water. Mice were inspected at least once every $24 \mathrm{~h}$. Two types of feed were provided, which included a normal rodent diet and a high-fat content diet that contained $15 \%$ fat from lard and was supplemented with $1.25 \%(\mathrm{w} / \mathrm{w})$ cholesterol.

Experimental groups. All 72 mice were fed a normal rodent diet for one week. Subsequently, mice were distributed randomly and evenly into an early-start (ES) and a late-start (LS) treatment group ( $\mathrm{n}=36$ each).

Mice in each group were randomly divided into four equal subgroups $(n=9)$ : Groups A (ES) and E (LS) were a normal diet control (NDC) group; groups B (ES) and F (LS) were a high-fat diet control (HDC) group; groups C (ES) and G (LS) were a low-dosage treatment (LDT) group receiving a high-fat diet and a low dosage of atorvastatin; and groups D (ES) and $\mathrm{H}$ (LS) were a high-dosage treatment (HDT) group receiving a high-fat diet and a high dosage of atorvastatin. Group $\mathrm{C}$ were orally administered $0.3 \mathrm{ml} / \mathrm{day}$ atorvastatin suspension, at a dosage of $5-\mathrm{mg} / \mathrm{kg}$ beginning in week 7 , whereas atorvastatin was administered to group $\mathrm{G}$ beginning in week 11. Group D were orally administered $0.3-\mathrm{ml} /$ day atorvastatin suspension, at a dosage of $10 \mathrm{mg} / \mathrm{kg}$, beginning in week 7 , whereas atorvastatin was administered to group $\mathrm{H}$ beginning in week 11 . Groups A, B, E, and F were administered an isodose of normal saline. Following 8 weeks of statin intervention, mice in the ES and LS groups were humanely sacrificed in weeks 15 and 19 , respectively.

Specimen harvesting. Mice were surgically anesthetized via intraperitoneal injection of $1 \%$ sodium pentobarbitone $(50 \mathrm{mg} / \mathrm{kg})$. The ascending aorta was removed from each mouse and immersed in $4 \%$ paraformaldehyde at room temperature, for $\geq 24 \mathrm{~h}$. Aortas were subsequently embedded in paraffin and cut transversally into serial sections 5- $\mu \mathrm{m}$ thick initiating at the aortic root. Remaining sections of the aortas (from the descending to the iliac aorta) were stored at $-70^{\circ} \mathrm{C}$.

Lipid detection. Serum harvested from the mice was analyzed to determine total triglyceride (TG) cholesterol (TC) and LDL-C levels, using an Olympus AU2700 High-Volume Chemistry-Immuno Analyzer (Olympus Corp., Tokyo, Japan).

Reverse transcription-quantitative polymerase chain reaction $(R T-q P C R)$ analysis. Total RNA was extracted from the frozen atherosclerotic carotid samples or THP-1 macrophages (American Type Culture Collection, Manassas, VA, USA) using RNAiso Plus reagent according to the manufacturer's protocol. Then, the total RNA was reverse-transcribed to cDNA using the PrimeScript ${ }^{\circledR}$ RT reagent kit with gDNA Eraser (RR047A) according to the manufacturer's protocol. Primer Express ${ }^{\circledR}$ software v3.0.1 (Applied Biosystems; Thermo Fisher Scientific, Inc.) and sequence information from the National Center for Biotechnology Information database (http://www.ncbi.nlm.nih.gov) were used to design the PCR primers for EMMPRIN, COX-2 and the housekeeping gene GAPDH. Primer sequences were as follows: EMMPRIN, forward 5'-GCAGAGGACACAGGCACTTAC-3' and reverse 5'-ACAGGCTCAGGAAGGAAGATG-3'; GAPDH, forward 5'-AGGTCGGTGTGAACGGATTTG-3' and reverse 5'-GGG GTCGTTGATGGCAACA-3'; COX-2, forward 5'-GATTGC CCGACTCCCTTGG-3' and reverse 5'-AACTGATGCGTG AAGTGCTG-3'. A total of $1 \mu \mathrm{g}$ cDNA was used as a template for Real-time PCR in 20- $\mu$ l reaction volumes with SYBR Premix Ex Taq II according to the manufacturer's instructions on a Stratagene Mx3000P qPCR system (Agilent Technologies, Inc., Santa Clara, CA, USA). The amplification protocol used was as follows: An initial denaturation step for $5 \mathrm{~min}$ at $95^{\circ} \mathrm{C}$ followed by 40 cycles of denaturation for $10 \mathrm{sec}$ at $95^{\circ} \mathrm{C}$, annealing for $30 \mathrm{sec}$ at $57^{\circ} \mathrm{C}$ and elongation for $30 \mathrm{sec}$ at $72^{\circ} \mathrm{C}$, and a final extension step at $72^{\circ} \mathrm{C}$ for $30 \mathrm{sec}$. Quantitative measurements were determined using the comparative $-2 \Delta \Delta \mathrm{Cq}$ method (18). All samples were normalized against the endogenous level of GAPDH. All results were repeated 
in triplicate and were analyzed using MxPro-Mx3000P software (Agilent Technologies, Inc.). The results for the relative expression levels were expressed as the mean \pm the standard deviation (SD).

Western blot analysis. For western blot analysis, mouse aortas were homogenized, ground in liquid nitrogen and subsequently lysed with radio-immunoprecipitation assay (RIPA) lysate and $100 \mu \mathrm{l}$ phenylmethylsulfonyl fluoride (PMSF) in ice for $30 \mathrm{~min}$. Following centrifugation at $20,000 \mathrm{x} g$ for $30 \mathrm{~min}$ at $4^{\circ} \mathrm{C}$, the protein was obtained from the deposition. Cells were washed twice with $1 \mathrm{ml}$ ice-cold phosphate-buffered saline (PBS) and lysed by adding $100 \mu \mathrm{l}$ ice-cold RIPA and $1 \mathrm{mmol} / \mathrm{l}$ PMSF. Protein concentration was determined using the Coomassie Brilliant Blue method with bovine serum albumin (BSA; $5 \mathrm{mg} / \mathrm{ml}$, Beyotime Institute of Biotechnology, Haimen, China) as a standard. Equal amounts (30 $\mu \mathrm{g} /$ well) of protein were separated by $10 \%$ SDS-PAGE and the protein was subsequently transferred, using electroblotting for $30 \mathrm{~min}$ at $50 \mathrm{~V}$, onto a polyvinylidene difluoride membrane. The membrane was blocked in a $6 \%$ non-fat milk solution in Tris-buffered saline with $0.5 \%$ Tween 20 (Santa Cruz Biotechnology, Inc.). The membrane was probed separately with a goat anti-EMMPRIN polyclonal antibody $(1: 1,000)$ at $4^{\circ} \mathrm{C}$ overnight, rabbit anti-goat HRP-conjugated secondary antibody $(1: 2,500)$ at $37^{\circ} \mathrm{C}$ for $1 \mathrm{~h}$, a rabbit polyclonal antibody against COX-2 $(1: 1,000)$ at $4^{\circ} \mathrm{C}$ overnight, goat anti-rabbit HRP-conjugated secondary antibody $(1: 2,000)$ at $37^{\circ} \mathrm{C}$ for $1 \mathrm{~h}$, rabbit anti-GAPDH polyclonal antibody $(1: 2,000)$ at $4^{\circ} \mathrm{C}$ overnight, then goat anti-rabbit HRP-conjugated secondary antibody $(1: 2,000)$ at $37^{\circ} \mathrm{C}$ for $1 \mathrm{~h}$. EMMPRIN, COX-2 and GADPH were detected using Pierce ${ }^{\mathrm{TM}}$ ECL Western Blotting Substrate (Thermo Fisher Scientific Inc., 32109) according to the manufacturer's protocol. Densitometric signals were quantified using ImageQuant TL 1.1 software (General Healthcare Bio-Sciences, Pittsburgh, PA, USA) and GAPDH was used as a loading control.

Immunology, histology, hematoxylin and eosin staining, and Sirius red staining. Following the rehydration and dewaxing of the serial 5- $\mu \mathrm{m}$ thick paraffin sections, they were incubated with $3 \%$ hydrogen peroxide to inhibit endogenous peroxidase activity. The sections were subsequently blocked with $5 \%$ BSA and were incubated for $30 \mathrm{~min}$ at $4^{\circ} \mathrm{C}$, prior to incubation overnight with the goat anti-EMMPRIN polyclonal antibody (1:500) in $1 \%(w / v)$ BSA in PBS. Sections were incubated at room temperature for $20 \mathrm{~min}$ with peroxidase-conjugated AffiniPure donkey anti-goat secondary antibodies (LI-COR Biosciences, IRDye $800 \mathrm{CW}, 1: 500$ ) in 1\% BSA in PBS. Hematoxylin staining was performed on the sections to reveal cell nuclei, using DAB as a substrate. Brown particles detected via light microscopy were considered positive. Image-Pro Plus 5.1 color microscopic image analysis software (Media Cybernetics, Inc., Rockville, MD, USA) was used to measure the positive staining integrated optical density (IOD) and provide semi-quantitative analysis. Five DAB-stained paraffin sections were randomly selected from each group and an average of five IOD measurements per group were calculated. Paraffin sections were immunostained for an rabbit anti-macrophage antibody (Abcam, Cambridge, MA,
USA, ab56297, $1: 100)$ at $4^{\circ} \mathrm{C}$ overnight and an anti- $\alpha$-actin antibody (Santa Cruz Biotechnology, sc-21078, 1:100) at $4^{\circ} \mathrm{C}$ overnight to confirm the presence of macrophages and smooth muscle cells (SMCs) in the vascular wall and plaques. Paraffin sections were deparaffinized, rehydrated and subsequently treated with $0.3 \%$ hydrogen peroxide in methanol for $30 \mathrm{~min}$ followed by $2 \%$ rabbit serum (sc-2338, Santa Cruz Biotechnology, Inc.) to abolish endogenous peroxidase activity and to block non-specific antibody binding, respectively. Sections were subsequently incubated overnight at $4^{\circ} \mathrm{C}$ with the rabbit anti-macrophage (sc-21078, 1:100) and anti- $\alpha$-actin antibody (ab56297, 1:100). Following incubation, the sections were washed several times with PBS and incubated for $30 \mathrm{~min}$ at $37^{\circ} \mathrm{C}$ with the goat anti-rabbit (IRDye680LT, 1:500) and donkey anti-goat (IRDye800CW, 1:500) secondary antibodies visualized with DAB Developer (OriGene Technologies, Inc.) and counterstained with $10 \%$ Mayer's hematoxylin. Subsequently, sections were mounted in Permamount and examined using light microscopy. Aortic paraffin sections were stained with hematoxylin and eosin (H\&E) and Sirius red.

Pathology and evaluation of plaque composition and lesion size. H\&E staining was performed on the aortic paraffin sections to observe the atherosclerotic plaque morphology. Computer-assisted morphometry (Image-Pro Plus 5.1; Media Cybernetics, Inc.) was used to analyze the cross-sectional area of each plaque, the relative plaque area (the ratio of the plaque area to the lumen area) and the fibrous cap thickness (FCT). The FCT was measured at 10 equidistant points around the cap of each slice. For each mouse, three sections (with an interval of 10 sections) from the thickest part of the plaque were selected and the mean value obtained was used for subsequent statistical analysis. Three consecutive paraffin sections from each mouse were used to detect macrophages and SMCs using an immunohistochemistry (IHC) assay, and to detect collagen using Sirius red staining. Lipoid vesicles were averaged directly from the H\&E sections. The vulnerability index (VI) of the plaque was calculated using the following formula (19): VI = (macrophages + lipoid vesicles) / (SMCs + collagen).

Cell culture. Human monocytic THP-1 macrophages were cultured in suspension in RPMI 1640 containing $100 \mathrm{U} / \mathrm{ml}$ penicillin, $10 \% \mathrm{FBS}$ and $100 \mu \mathrm{g} / \mathrm{ml}$ streptomycin at $37^{\circ} \mathrm{C}$ in an atmosphere containing $5 \% \mathrm{CO}_{2}$. A total of $1 \times 10^{6} \mathrm{THP}-1$ cells per well were seeded in 6-well plates and stimulated with $0.1 \mu \mathrm{m}$ PMA for $48 \mathrm{~h}$ until they adhered to the wells and exhibited macrophage-like morphology. Plates were washed twice with $1 \mathrm{ml}$ PBS following culture. Cells were cultured in serum-free medium for an additional $24 \mathrm{~h}$ and incubated with the corresponding stimuli: $100-\mu \mathrm{m} / \mathrm{ml}$ ox-LDL was used to induce COX-2 and EMMPRIN expression and no ox-LDL was used as a control. For inhibition experiments, the cells were pre-incubated with $5 \mu \mathrm{m}$ atorvastatin, $5 \mu \mathrm{m}$ ezetimibe or $10 \mu \mathrm{m} \mathrm{NS}-398$ for $1 \mathrm{~h}$.

PGE2 assay. A PGE2 ELISA kit (EIA-1112) was used to measure PGE2 levels in the culture medium, according to the manufacturer's protocol. Absorbance was measured at 
$405 \mathrm{~nm}$ with a microplate reader (Thermo Fisher Scientific Inc. Waltham, MA, USA).

Statistical analysis. Image-Pro Plus 5.1 software was used to count gray-scale and intensity values, and areas of interest. SPSS 13.0 software (SPSS, Inc., Chicago, IL, USA) was used for statistical analyses. The data were presented as the mean \pm the SD of three independent measurements. Groups of data were compared by performing one-way analysis of variance, followed by Tukey's multiple comparison tests. Pearson's product-moment correlation coefficient was used to analyze linear correlations. $\mathrm{P}<0.05$ was considered to indicate a statistically significant difference.

\section{Results}

Lipid levels. Under the high-fat feeding conditions, there were no statistically significant differences observed in TC, LDL-C and TG levels among the three groups of $\mathrm{ApoE}^{-1 /}$ mice.

High-fat diet leads to VPs in the aortas of Apo $E^{-/-}$mice. Plaque in the HDC groups (groups B and F) was characterized by thin fibrous caps, extensive acellular collagenous masses and large lipoid vesicles that extended to and narrowed the lumen under the microscope. By contrast, the plaque in the NDC groups (groups A and E) contained thick fibrous caps and small lipoid vesicles (Fig. 1A). The relative plaque area in the HDC groups was significantly larger than that of the NDC groups $(\mathrm{P}<0.01$; Fig. $1 \mathrm{~B})$. VP was defined as an FCT $<65 \mathrm{~mm}(20,21)$. The mean FCT in the HDC groups was $<65 \mathrm{~mm}$ and was significantly lower than that of the NDC groups $(\mathrm{P}<0.05$; Fig. 1C). The macrophages and the SMCs in the plaque were stained brown during the IHC assay. Sirius Red stained type I collagen yellow and red, and type III collagen green (Fig. 1A). The HDC groups exhibited significantly increased percentages of macrophages $(\mathrm{P}<0.05)$ and lipoid vesicles $(\mathrm{P}<0.01)$, and decreased percentages of SMCs $(\mathrm{P}<0.05)$ and collagen $(\mathrm{P}<0.01)$ in plaques compared with the NDC groups (Fig. 1D). Therefore, the HDC groups were classified as having VP. Mice in the HDC groups exhibited decreased FCT $(\mathrm{P}<0.05$; Fig. 1C) and increased VI $(\mathrm{P}<0.01$; Fig. 1E). A positive histological correlation was detected between EMMPRIN expression and the VI $(\mathrm{P}<0.05$; Fig. $1 \mathrm{~F})$.

High-fat diet increases EMMPRIN expression in the VPs of ApoE ${ }^{-/}$mice. The results of the RT-qPCR (Fig. 2), the western blot analysis (Fig. 3) and the IHC assay (Fig. 4) revealed significantly increased EMMPRIN expression in the HDC groups compared with the NDC $(\mathrm{P}<0.01)$, LDT (ES, $\mathrm{P}<0.05$; LS, $\mathrm{P}<0.01)$ and HDT $(\mathrm{P}<0.01)$ groups, in ES and LS rats. The significant difference between the HDC and NDC groups indicates that EMMPRIN expression was higher in VPs than in stable plaques.

Atorvastatin decreases EMMPRIN expression in the aortas and plaques of ApoE ${ }^{-1-}$ mice. Significantly decreased expression of EMMPRIN was observed in the LDT (ES, $\mathrm{P}<0.05$; LS, $\mathrm{P}<0.01)$ and HDT $(\mathrm{P}<0.01)$ groups compared with the HDC group, according to the mRNA (Fig. 2D-F) and protein levels (Fig. 3B, E and F). This was confirmed by the histological findings shown in Fig. 4 (Fig. 4A, C and D). Histological EMMPRIN expression was positively correlated with the VI $(\mathrm{P}<0.01$; Fig. 4F).

High dose atorvastatin induced greater downregulation of EMMPRIN expression than the low dose $(\mathrm{P}<0.05)$. EMMPRIN expression was evaluated using RT-qPCR (Fig. 2F), western blotting (Fig. 3B and D) and an IHC assay (Fig. 4A and E). In the HDT and LDT groups, EMMPRIN expression was significantly lower in the ES than in the LS group, according to mRNA (LDT, P<0.05; HDT, P<0.01; Fig. $2 \mathrm{~F}$ ) and protein levels $(\mathrm{P}<0.05$; Fig. $3 \mathrm{~B}$ and $\mathrm{D})$. The histological findings also indicated significantly lower EMMPRIN expression in the ES HDT group than in the corresponding LS group $(\mathrm{P}<0.05$; Fig. 4E).

$O x-L D L$ induces the secretion of $P G E 2$ and the expression of COX-2 and EMMPRIN in THP-1 macrophages. Treatment of THP-1 macrophages with $100 \mu \mathrm{g} / \mathrm{ml}$ ox-LDL for $6 \mathrm{~h}$ induced a rapid increase in PGE2 secretion, which peaked at $12 \mathrm{~h}$ (Fig. 5A). RT-qPCR and western blotting were used to examine whether COX-2 and EMMPRIN mRNA and protein expression were upregulated in THP-1 macrophages stimulated with ox-LDL in vitro. The mRNA and protein levels of COX-2 and EMMPRIN were increased significantly at $6 \mathrm{~h}$ compared with the levels in the control group $(\mathrm{P}<0.05$; Fig. $5 \mathrm{~B}$ and $\mathrm{C})$. A positive correlation was observed between changes in mRNA and protein expression of EMMPRIN and COX-2 (data not shown).

Atorvastatin inhibits ox-LDL-induced expression of COX-2 and EMMPRIN in THP-1 macrophages. Following pretreatment with atorvastatin or ezetimibe (both $5 \mu \mathrm{M}$ ) for $1 \mathrm{~h}$, THP-1 macrophages were stimulated with $100 \mu \mathrm{g} / \mathrm{ml}$ ox-LDL for an additional $12 \mathrm{~h}$. COX-2 and EMMPRIN protein expression levels were subsequently measured using western blotting. Atorvastatin markedly inhibited the ox-LDL-induced expression of COX-2 and EMMPRIN, whereas ezetimibe did not (Fig. 5D). These results suggest that the upregulation of EMMPRIN expression may be associated with increased COX-2 levels in THP-1 macrophages.

Additionally, the results demonstrated that ox-LDL was able to markedly upregulate COX-2 and EMMPRIN expression; however, further research on the role of the COX-2/PGE2 pathway is required. Therefore, these findings elucidate the possible effect of COX-2/PGE2 on ox-LDL-induced EMMPRIN expression.

Following pretreatment with $5 \mu \mathrm{M}$ atorvastatin or $10 \mu \mathrm{M}$ NS-398 for $1 \mathrm{~h}$, THP-1 macrophages were stimulated with $1 \mu \mathrm{M}$ ox-LDL or $0.1 \mu \mathrm{M}$ ox-LDL and PGE2, for a further $12 \mathrm{~h}$. Notably, when atorvastatin or NS-398 was used as a co-pretreatment with PGE2, the inhibitory effects of atorvastatin or NS-398 on ox-LDL-induced EMMPRIN mRNA and protein expression were significantly reversed $(\mathrm{P}<0.05$; Fig. 5E and F). Furthermore, PGE2 levels were significantly increased by ox-LDL $(\mathrm{P}<0.05)$ and decreased by atorvastatin and NS-398 (Fig. 5G). These results indicate that EMMPRIN expression in THP-1 macrophages was elevated due to an increase in PGE2 levels, whereas atorvastatin and NS-398 each suppressed PGE2 expression. This inhibition was reversed following the addition of PGE2. 

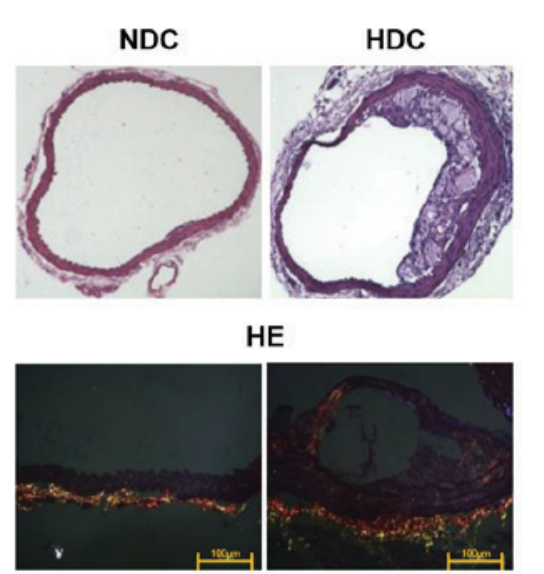

Collagen

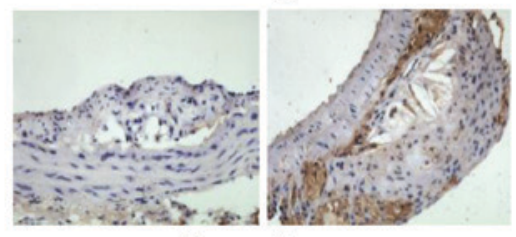

Macrophages

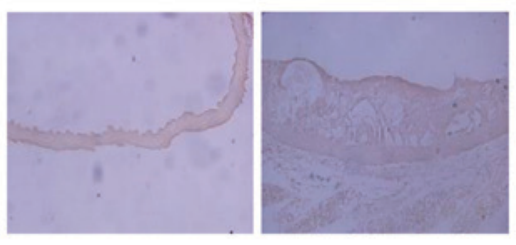

SMC

B

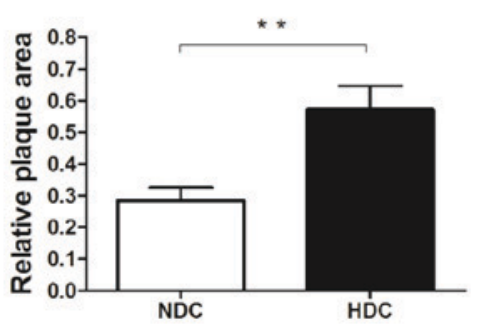

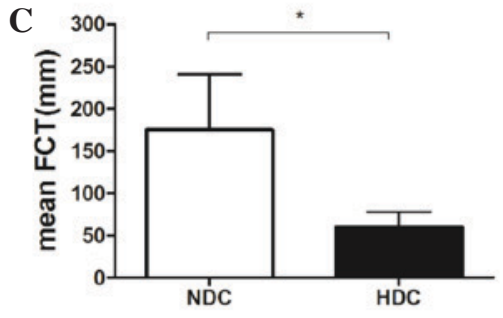

D

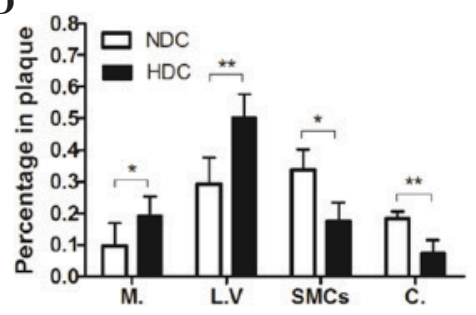

E

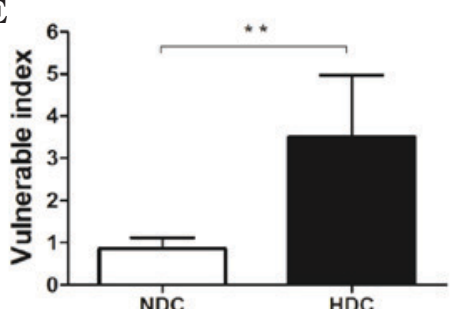

F

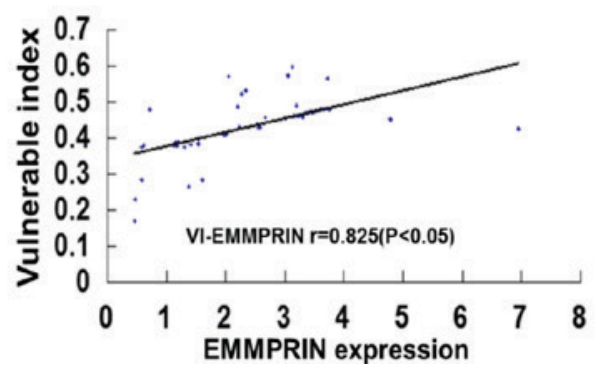

Figure 1. Morphology and VI of the aortic plaques of $\mathrm{ApoE}^{-/}$male mice in the early-start groups ( $\mathrm{n}=9$ ). (A) HE staining of the ascending aortas from $\mathrm{ApoE}^{-1}$ male mice in the NDC and HDC groups (magnification, x100). Type I collagen is stained yellow and red, and type III collagen is stained green (magnification, x400; scale bar, $100 \mu \mathrm{m}$ ). Macrophages and SMCs are stained brown. (B) Relative plaque area was significantly increased in the HDC compared with the NDC group. (C) Average FCT was significantly decreased in the HDC compared with the NDC group. (D) Percentages of Ms and LVs were increased and percentages of SMCs and $\mathrm{C}$ were decreased in plaques in the HDC group compared with the NDC group. (E) VI was significantly increased in the HDC compared with the NDC group. (F) There was a positive correlation between histological expression of EMMPRIN and VI. " $\mathrm{P}<0.05$; ${ }^{* *} \mathrm{P}<0.01$. VI, vulnerability index; $\mathrm{ApoE}^{-1}$, apolipoprotein E knockout; HE, hemotoxylin and eosin; SMC, smooth muscle cell; NDC, normal diet control; HDC, high-fat diet control; FCT, fibrous cap thickness; M, macrophage; LV, lipoid vesicle; C, collagen; EMMPRIN, extracellular matrix metalloproteinase inducer; r, Pearson's product-moment correlation coefficient.

\section{Discussion}

In the present study, the increased EMMPRIN expression in an $\mathrm{ApoE}^{-/-}$atherosclerotic mouse model and the effect of atorvastatin treatment on EMMPRIN expression was demonstrated. Atorvastatin treatment reduced artery atherosclerotic lesion progression and EMMPRIN expression. The effect of atorvastatin was associated with the COX-2/PGE2 signaling pathway. These data implicate EMMPRIN as a harmful factor in the development of VPs and indicate that atorvastatin inhibits the expression of EMMPRIN in plaque.

EMMPRIN is a 58-kDa cell surface glycoprotein in the immunoglobulin superfamily (22). Previous experimental research has demonstrated that EMMPRIN is localized in macrophage-rich regions of human atherosclerotic plaques (8) and clinical researchers have previously demonstrated that EMMPRIN is upregulated in monocytes in acute myocardial infarction (AMI), whereas it is not in patients with stable 
A

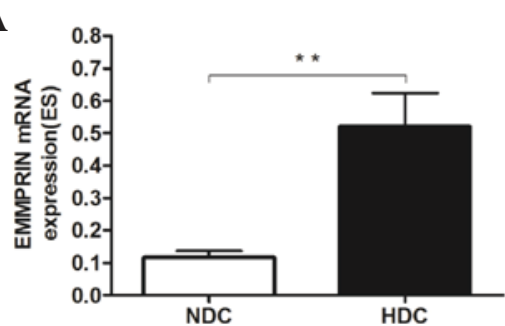

$\mathbf{D}$

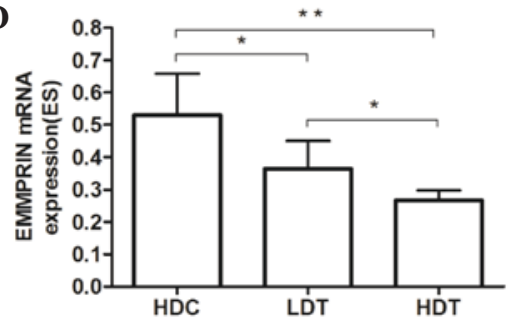

B
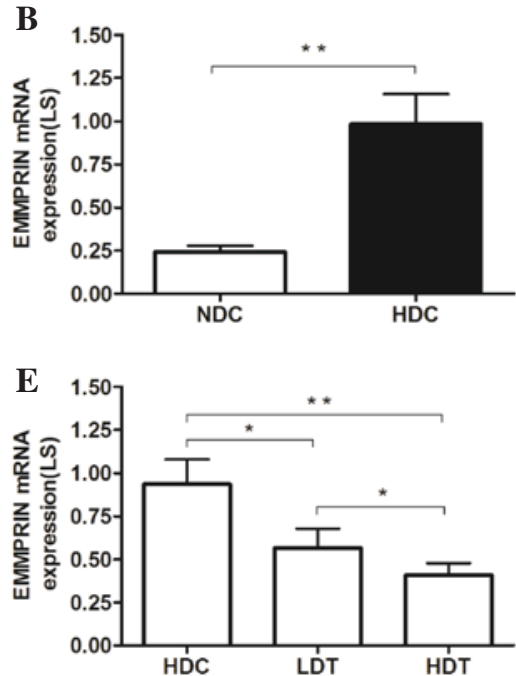
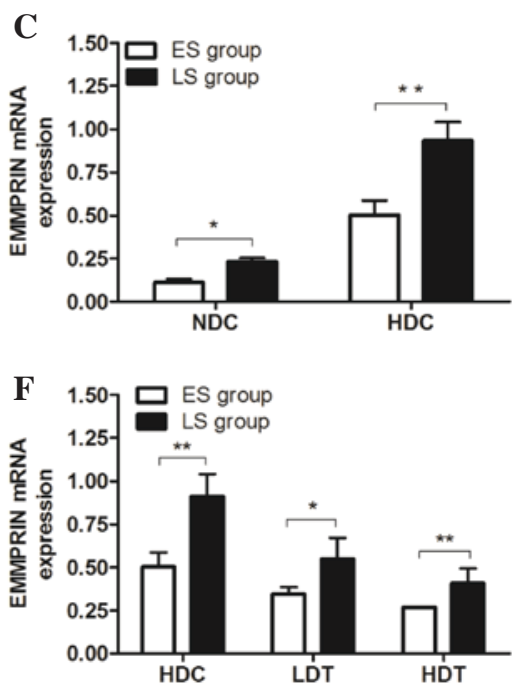

Figure 2. mRNA expression in the aortas of $\mathrm{ApoE}^{-/-}$male mice was determined by reverse transcription-quantitative polymerase chain reaction. (A-C) For the ES and LS groups, the mRNA expression of EMMPRIN in the HDC group was significantly higher than that of the NDC group. In the (D) ES and (E) LS groups, mRNA expression of EMMPRIN in the HDC group was significantly higher than that of the LDT and HDT groups. Furthermore, EMMPRIN mRNA expression in the HDT group was significantly lower than that of the LDT group. (F) EMMPRIN mRNA expression in the ES group was significantly lower than that of the corresponding LS group in the HDC, LDT, and HDT subgroups. ${ }^{*} \mathrm{P}<0.05 ;^{* *} \mathrm{P}<0.01$. ApoE ${ }^{-/}$, apolipoprotein E knockout; ES, early-start; LS, late-start; EMMPRIN, extracellular matrix metalloproteinase inducer; HDC, high-fat diet control; NDC, normal diet control; LDT, low-dose atorvastatin treatment; HDT, high-dose atorvastatin treatment.

A

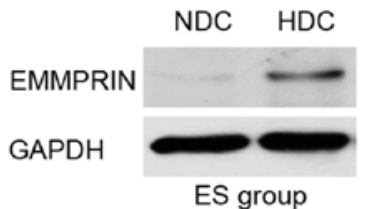

C

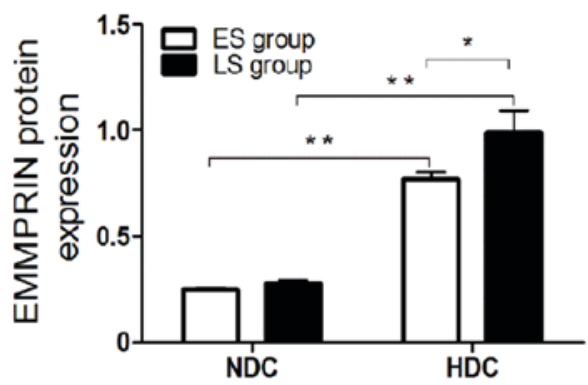

E

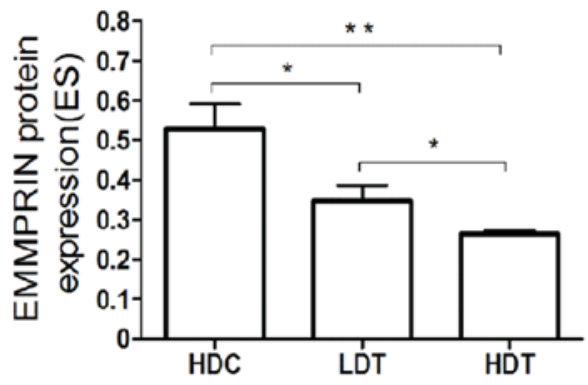

B

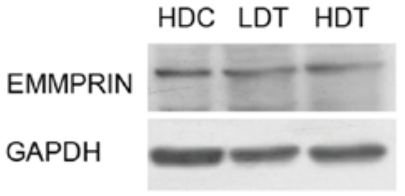

ES group

LS group

D

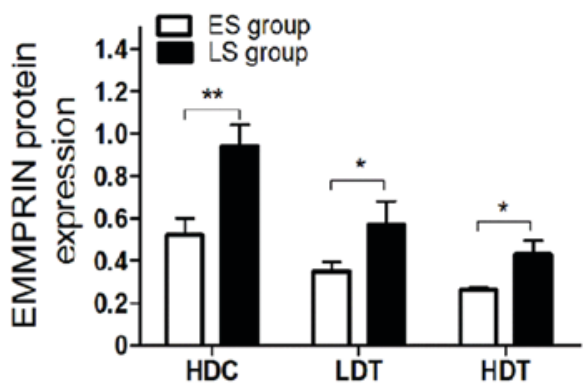

F

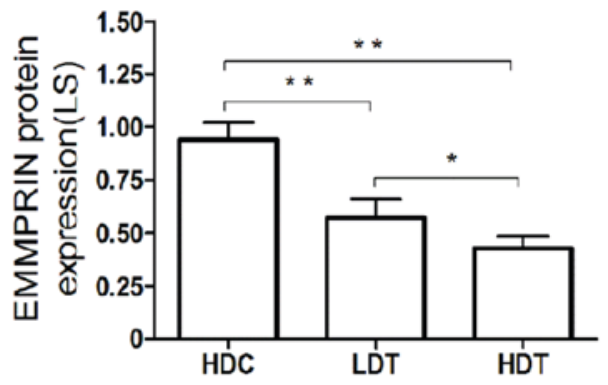

Figure 3. (A and B) Protein expression in the aortas of $\mathrm{ApoE}^{-/-}$male mice was determined via western blot analyses. For the ES and LS groups, (C) the protein expression of EMMPRIN in the HDC group was higher than that of the NDC group. (D) EMMPRIN protein expression in the ES group was significantly lower than in the corresponding LS group in the HDC, LDT and HDT subgroups. EMMPRIN protein expression was significantly higher in the HDC group than in the LDT and HDT groups, in (E) ES and (F) LS mice. Furthermore, EMMPRIN expression was significantly higher in the LDT than in the HDT group in ES and LS mice. ${ }^{*} \mathrm{P}<0.05 ;{ }^{* *} \mathrm{P}<0.01$. ApoE ${ }^{-/}$, apolipoprotein E knockout; ES, early-start; LS, late-start; EMMPRIN, extracellular matrix metalloproteinase inducer; HDC, high-fat diet control; NDC, normal diet control; LDT, low-dose atorvastatin treatment; HDT, high-dose atorvastatin treatment; GAPDH, glyceraldehyde 3-phosphate dehydrogenase. 
A

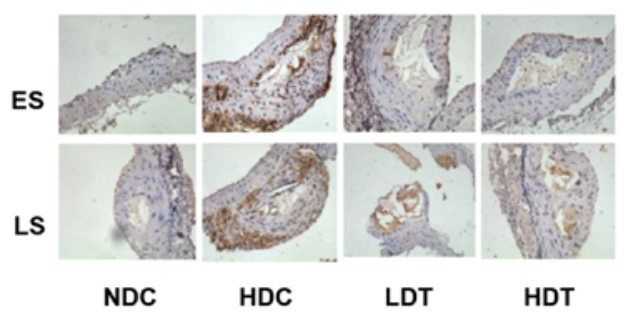

C

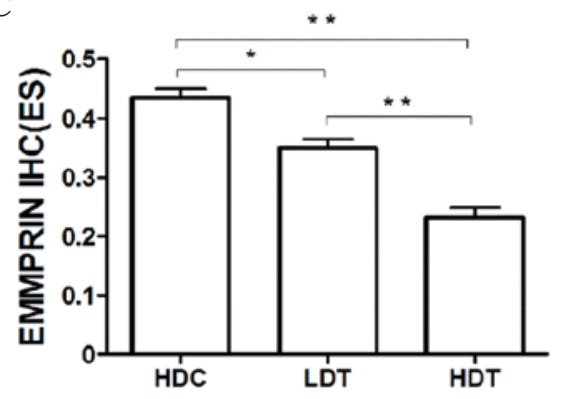

E

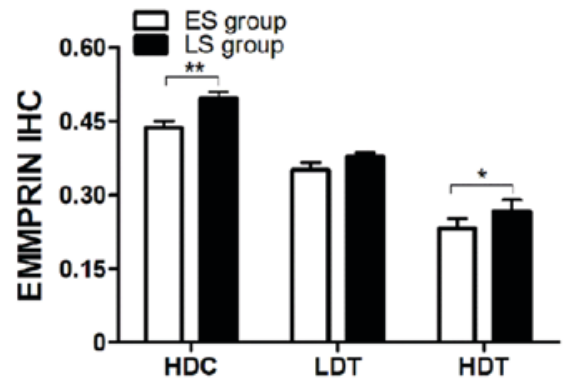

B

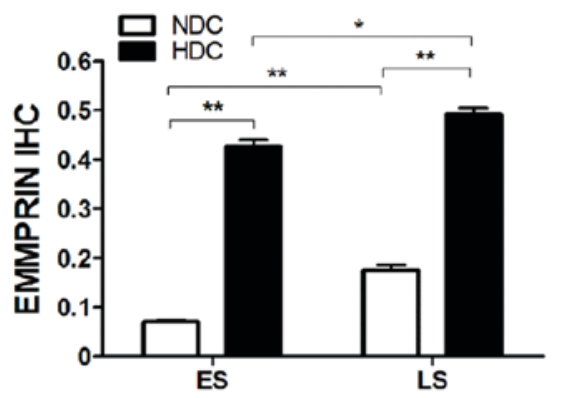

D

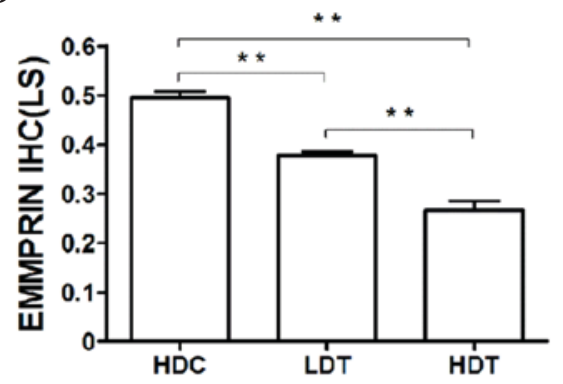

F

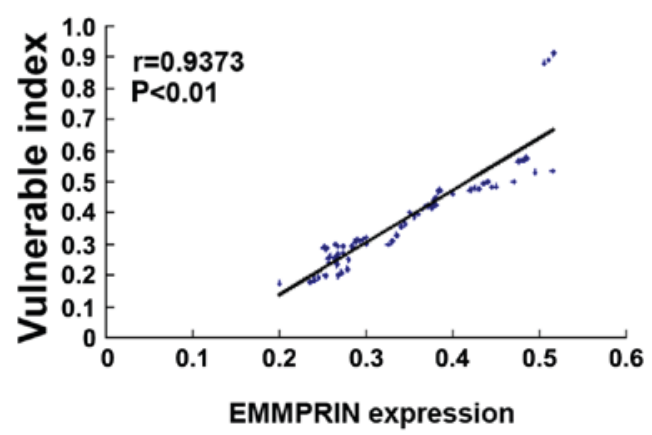

Figure 4. IHC analysis of EMMPRIN in the aortas of ApoE ${ }^{-/-}$male mice in the four different groups (n=9). (A) Microscopic images (magnification, $\mathrm{x} 400$ ) present the IHC results. The brown color represents a positive result. (B) In the ES and LS groups, EMMPRIN histological expression in the HDC group was significantly higher than in the NDC group. Furthermore, EMMPRIN histological expression was significantly higher in LS subgroups compared with the corresponding ES subgroups. In the (C) ES and (D) LS groups, EMMPRIN histological expression was significantly lower in the HDT group than in the LDT group and was significantly lower in the LDT and HDT groups compared with the HDC group. (E) EMMPRIN histological expression in the LS group was significantly higher in the HDC and HDT groups compared with the corresponding ES subgroups. (F) VI was positively correlated with the histological expression of EMMPRIN. ${ }^{*} \mathrm{P}<0.05 ;{ }^{* *} \mathrm{P}<0.01$. IHC, immunohistochemical; EMMPRIN, extracellular matrix metalloproteinase inducer; ApoE ${ }^{-/}$, apolipoprotein E knockout; ES, early-start; LS, late-start; HDC, high-fat diet control; NDC, normal diet control; HDT, high-dose atorvastatin treatment; LDT, low-dose atorvastatin treatment; VI, vulnerability index; r, Pearson's product-moment correlation coefficient.

angina (23). Therefore, EMMPRIN may serve a role in the destabilization of atheroma by inducing MMP-mediated ECM degradation (24). The present study demonstrated the increased infiltration of macrophages into VPs, in which EMMPRIN was highly expressed. Macrophages secrete MMPs, which promote the progression of VPs by degrading the ECM. It has been suggested that nuclear factor $\kappa \mathrm{B}(\mathrm{NF}-\kappa \mathrm{B})$ is a critical promoter of MMPs $(25,26)$. Therefore, downregulating EMMPRIN expression in macrophages may reduce the potential damage caused by MMPs via the NF- $\kappa$ B pathway and inhibit the development of VPs.

ApoE $^{-/-}$mice contain the entire spectrum of lesions that typically occur during atherogenesis and this mouse model was the first to develop lesions similar to those observed in humans (27). Previous studies have demonstrated that feeding
$\mathrm{ApoE}^{-/-}$mice either chow or a high fat, Western-type diet may lead to plaque rupture $(4,28-30)$. In one such study, the brachiocephalic arteries of $62 \%$ of mice exhibited acutely ruptured plaques after 8 weeks of feeding either normal chow or a high-fat diet (29). Fragmentation and the loss of SMCs and elastin in the fibrous caps were observed in relatively small and lipid-rich plaques that overlay large complex lesions, characterizing the rupture (4).

In the present study, lesions were immunostained to identify macrophages, collagen, SMCs and the percentage of the lesional area to determine the lesion composition in the plaques. Apoptosis of vascular SMCs and macrophages may promote pro-coagulation, plaque growth and rupture, which are the major consequences of atherosclerosis in humans (31). Results from previous studies have suggested that preventing 
A

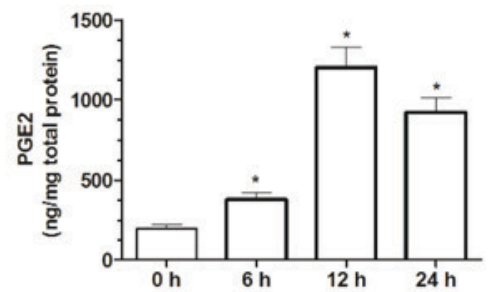

C
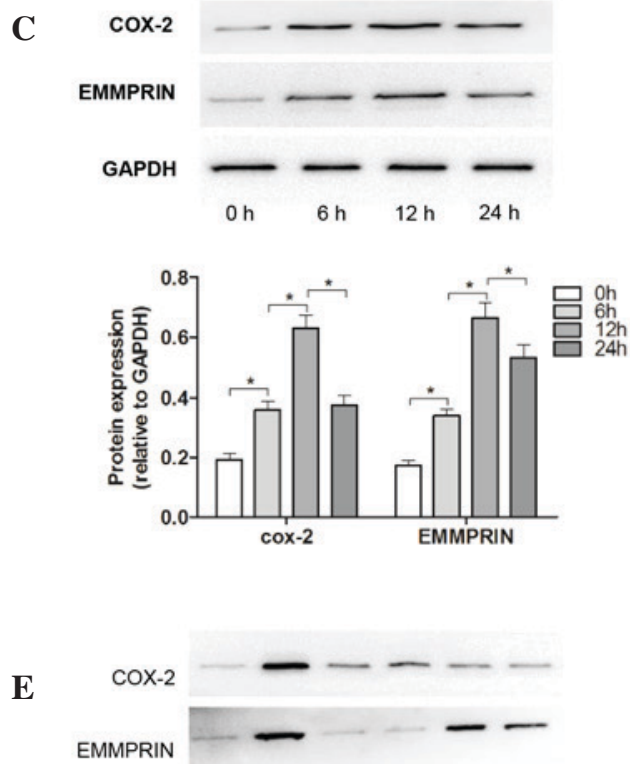

$\mathrm{GAPDH}----\cdots$
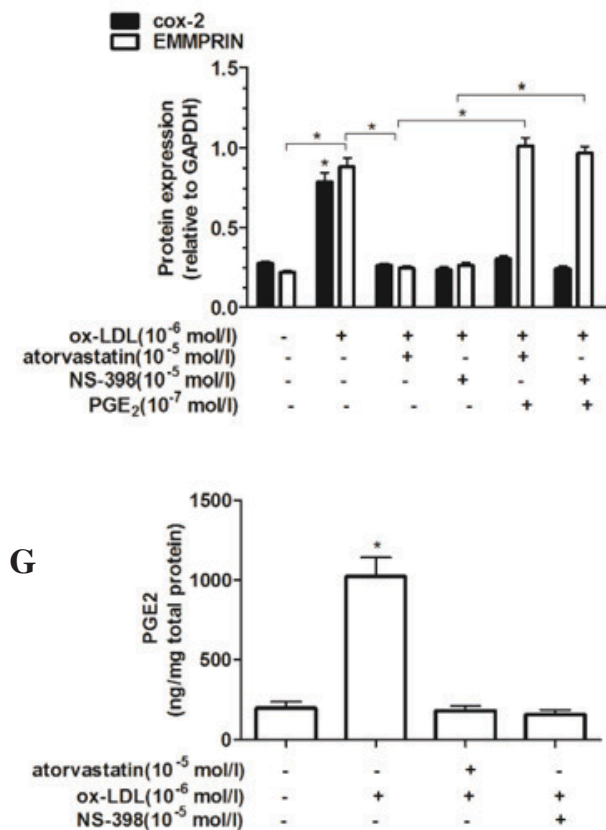

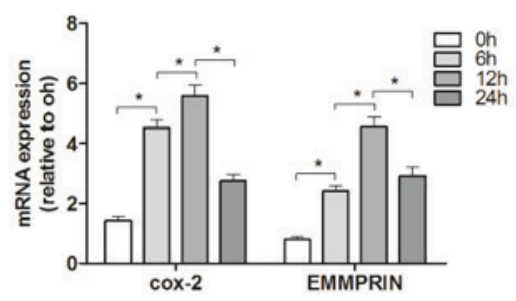

D

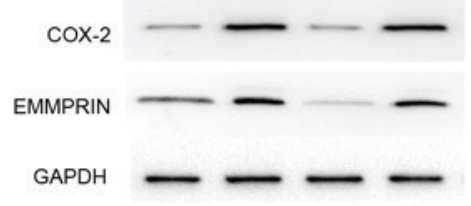

cox-2

EMMPRIN

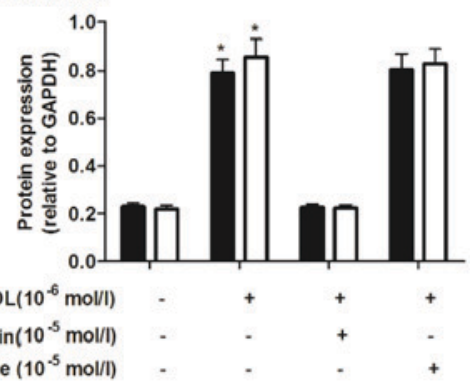

$\operatorname{cox}-2$

EMMPRIN

F

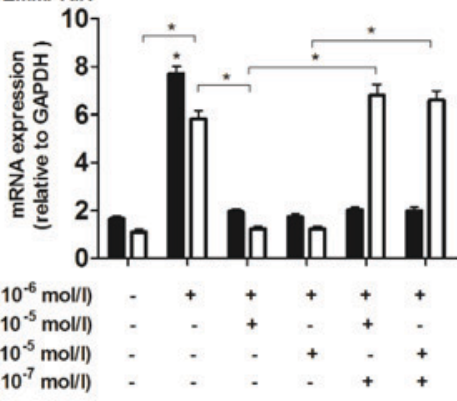

Figure 5. Atorvastatin inhibits ox-LDL-induced EMMPRIN expression in THP-1 macrophages via the COX-2/PGE2 pathway. (A) Ox-LDL significantly increases PGE2 expression in THP-1 macrophages. THP-1 macrophages were treated with $100 \mu \mathrm{g} / \mathrm{ml}$ ox-LDL and PGE2 expression was measured using ELISA. (B) mRNA expression of COX-2 and EMMPRIN was measured by RT-qPCR. (C) COX-2 and EMMPRIN protein expression was measured via western blotting. 6 h: $\mathrm{P}<0.05$ vs. 0 h, 12 h: $\mathrm{P}<0.05$ vs. 6, and 24 h: $\mathrm{P}<0.05$ vs. 12 h. (D) Atorvastatin inhibited ox-LDL-induced COX-2 and EMMPRIN expression in THP-1 macrophages. Cells were pretreated with $5 \mu \mathrm{M}$ atorvastatin or $5 \mu \mathrm{M}$ ezetimibe for $1 \mathrm{~h}$, followed by stimulation with $100 \mu \mathrm{g} / \mathrm{ml}$ ox-LDL for an additional $12 \mathrm{~h}$. Western blotting was subsequently performed. PGE2 reversed the inhibitory effects of atorvastatin or NS-398 on ox-LDL-induced EMMPRIN expression. Cells were pretreated with atorvastatin or ezetimibe (both $5 \mu \mathrm{M}$ ) for $1 \mathrm{~h}$, followed by stimulation with $100 \mu \mathrm{g} / \mathrm{ml}$ ox-LDL or ox-LDL and PGE2 for an additional $12 \mathrm{~h}$. The mRNA and protein expression of EMMPRIN and COX-2 were determined via (E) western blotting and (F) RT-qPCR, and the amount of PGE2 in the medium was measured via (G) ELISA. "P<0.05. Data are presented as the mean \pm standard error of the mean. ox-LDL; oxidized low-density lipoprotein; EMMPRIN, extracellular matrix metalloproteinase inducer; COX-2, cyclooxygenase-2; PGE2, prostaglandin E2; RT-qPCR, reverse transcription quantitative polymerase chain reaction. 
ECM degradation or increasing ECM components, including elastin and collagen, may decrease the risk of plaque progression, thereby improving outcomes in atherosclerotic disease (32-34). Therefore, the present study evaluated the stability of the plaques (the VI). The results indicated that EMMPRIN was increased in VPs with a high ratio of macrophages to lipoid vesicles and in VPs with a low ratio of SMCs to collagen, which suggests a latent link between EMMPRIN and these plaque compositions. Previous studies have indicated that EMMPRIN is upregulated during monocyte differentiation into macrophages and that foam cells induce the proliferation and migration of SMCs $(8,35,36)$. This suggests that EMMPRIN may induce VPs through these pathways.

Atorvastatin is an inhibitor of $\mathrm{HMG}-\mathrm{CoA}$ reductase and is widely used to treat patients with atherosclerosis. Atorvastatin was selected for the current study due to its anti-atherosclerotic effect. Following confirmation of the correlation between EMMPRIN and VP, atorvastatin was used as an intervention in $\mathrm{ApoE}^{-/}$mice that were fed a high-fat diet. The results suggested that atorvastatin was able to downregulate EMMPRIN expression in the aortas of $\mathrm{ApoE}^{-/-}$mice, according to mRNA and protein levels and histological findings. Additionally, artery atherosclerotic lesion progression was inhibited. In the present study, ApoE ${ }^{-/-}$mice were 23 weeks old when the experiment in the ES group was completed. The treatment subgroups had been treated with atorvastatin for 8 weeks, whereas treatment of LS group mice had been initiated later and lasted for 4 weeks. The results demonstrated that plaque VI and EMMPRIN expression were significantly greater in the LS group. This suggests that differences in the initiation time and duration of the statin intervention may have affected plaque vulnerability and that the difference observed following early statin treatment may be due to the early reduction in the expression of EMMPRIN during SMC migration and macrophage aggregation.

A number of studies have indicated that EMMPRIN levels may be increased by various stimuli, including free radicals, cytokines and ox-LDL, that have key regulatory roles in MMP activity $(37,38)$. MMPs make an essential contribution to the pathophysiology of atherosclerosis $(23,39,40)$. A link has been identified between the presence of membrane type 1-MMP, MMP-2 and MMP-9 within vascular walls and unstable plaque phenotypes that are prone to rupture (41-43). In cardiac disease, patients with AMI exhibit increased EMMPRIN, MMP-2 and MMP-9 levels, whereas patients with stable angina do not (44). In unstable plaques, levels of MMP-9 activity are increased, whereas in stable plaques, levels of MMP-2 activity levels are increased (45). MMP expression levels are associated with different glycosylated EMMPRIN forms (23). Additionally, different EMMPRIN forms have been observed among different plaque phenotypes (46). The present data suggested that atorvastatin may have prevented the process of atherosclerosis and plaque rupture by reducing EMMPRIN expression in plaques. Atorvastatin had no significant effect on the plasma lipid and cholesterol concentrations in the $\mathrm{ApoE}^{-/-}$mice, which Bea et al (47) and Johnson et al (29) have previously documented for simvastatin and pravastatin, respectively. Atorvastatin may have a pleiotropic effect, similar to other statins (48).
In previous studies conducted by the present authors $(49,50)$, it was demonstrated that angiotensin II upregulated EMMPRIN expression in macrophages via the COX-2 pathway. Following the finding that atorvastatin induced COX-2 expression in macrophages and monocytes, the mechanism used in the atorvastatin-induced inhibition of EMMPRIN expression in $\mathrm{ApoE}^{-/-}$mice was investigated. In an in vitro study, it was demonstrated that the expression of the COX-2 gene and protein induced by ox-LDL were also inhibited by atorvastatin and that PGE2 restored the EMMPRIN inhibition induced by reductase $\mathrm{HMG}-\mathrm{CoA}$ inhibitors or COX-2 inhibitors. These observations indicated that atorvastatin was able to inhibit ox-LDL-mediated upregulation of EMMPRIN expression via the COX-2/PGE2 signaling pathway.

In conclusion, the present study evaluated EMMPRIN expression in aortic plaques in $\mathrm{ApoE}^{-/-}$mice according to dietary fat content. It was demonstrated that EMMPRIN expression was upregulated and was positively correlated with the development of VPs. Atorvastatin treatment was able to reduce atherosclerotic plaque vulnerability by downregulating EMMPRIN expression. This inhibitory effect of atorvastatin on EMMPRIN may occur via the COX-2/PGE2 signaling pathway. Further studies are necessary to confirm that this is the case.

\section{References}

1. Aronson D and Edelman ER: Coronary artery disease and diabetes mellitus. Cardiol Clin 32: 439-455, 2014.

2. Pagidipati NJ and Peterson ED: Acute coronary syndromes in women and men. Nat Rev Cardiol 13: 471-480, 2016.

3. Lusis AJ: Atherosclerosis. Nature 407: 233-241, 2000.

4. Rosenfeld ME, Polinsky P, Virmani R, Kauser K, Rubanyi G and Schwartz SM: Advanced atherosclerotic lesions in the innominate artery of the ApoE knockout mouse. Arterioscler Thromb Vasc Biol 20: 2587-2592, 2000.

5. Schonbeck U and Libby P: CD40 signaling and plaque instability. Circ Res 89: 1092-1103, 2001.

6. Rajagopalan S, Meng XP, Ramasamy S, Harrison DG and Galis ZS: Reactive oxygen species produced by macrophage-derived foam cells regulate the activity of vascular matrix metalloproteinases in vitro. Implications for atherosclerotic plaque stability. J Clin Invest 98: 2572-2579, 1996.

7. Fabunmi RP, Moore KJ, Libby $\mathrm{P}$ and Freeman MW: Stromelysin-1 (MMP-3) expression driven by a macrophage-specific promoter results in reduced viability in transgenic mice. Atherosclerosis 148: 375-386, 2000.

8. Major TC, Liang L, Lu X, Rosebury W and Bocan TM: Extracellular matrix metalloproteinase inducer (EMMPRIN) is induced upon monocyte differentiation and is expressed in human atheroma. Arterioscler Thromb Vasc Biol 22: 1200-1207, 2002.

9. Patti G, Pasceri V, Colonna G, Miglionico M, Fischetti D, Sardella G, Montinaro A and Di Sciascio G: Atorvastatin pretreatment improves outcomes in patients with acute coronary syndromes undergoing early percutaneous coronary intervention: Results of the ARMYDA-ACS randomized trial. J Am Coll Cardiol 49: 1272-1278, 2007.

10. Soeda T, Uemura S, Okayama S, Kawakami R, Sugawara Y, Nakagawa H, Matsumoto T, Sung JH, Nishida T, Senoo A, et al: Intensive lipid-lowering therapy with rosuvastatin stabilizes lipid-rich coronary plaques. -Evaluation using dual-source computed tomography. Circ J 75: 2621-2627, 2011.

11. Murphy SA, Cannon CP, Wiviott SD, de Lemos JA, Blazing MA, McCabe CH, Califf RM and Braunwald E: Effect of intensive lipid-lowering therapy on mortality after acute coronary syndrome (a patient-level analysis of the Aggrastat to Zocor and Pravastatin or Atorvastatin Evaluation and Infection Therapy-Thrombolysis in Myocardial Infarction 22 trials). Am J Cardiol 100: 1047-1051, 2007. 
12. Farmer JA and Gotto AM Jr: Currently available hypolipidaemic drugs and future therapeutic developments. Baillieres Clin Endocrinol Metab 9: 825-847, 1995.

13. Illingworth DR and Bacon S: Hypolipidemic effects of HMG-CoA reductase inhibitors in patients with hypercholesterolemia. Am J Cardiol 60: 33G-42G, 1987.

14. O'Driscoll G, Green D and Taylor RR: Simvastatin, an HMG-coenzyme A reductase inhibitor, improves endothelial function within 1 month. Circulation 95: 1126-1131, 1997.

15. Stroes ES, Koomans HA, de Bruin TW and Rabelink TJ: Vascular function in the forearm of hypercholesterolaemic patients off and on lipid-lowering medication. Lancet 346: 467-471, 1995

16. Meredith IT, Plunkett JC, Worthley SG, Hope SA and Cameron JD: Systemic inflammatory markers in acute coronary syndrome: Association with cardiovascular risk factors and effect of early lipid lowering. Coron Artery Dis 16: 415-422, 2005.

17. Ridker PM: Inflammation, infection, and cardiovascular risk: How good is the clinical evidence? Circulation 97: 1671-1674, 1998.

18. Livak KJ and Schmittgen TD: Analysis of relative gene expression data using real-time quantitative PCR and the 2(-Delta Delta C(T)) Method. Methods 25: 402-408, 2001.

19. Williams H, Johnson JL, Carson KG and Jackson CL: Characteristics of intact and ruptured atherosclerotic plaques in brachiocephalic arteries of apolipoprotein E knockout mice. Arterioscler Thromb Vasc Biol 22: 788-792, 2002.

20. Burke AP, Farb A, Malcom GT, Liang YH, Smialek J and Virmani R: Coronary risk factors and plaque morphology in men with coronary disease who died suddenly. N Engl J Med 336: 1276-1282, 1997.

21. Virmani R, Burke A and Farb A: Coronary risk factors and plaque morphology in men with coronary disease who died suddenly. Eur Heart J 19: 678-680, 1998.

22. Biswas C, Zhang Y, DeCastro R, Guo H, Nakamura T, Kataoka $\mathrm{H}$ and Nabeshima K: The human tumor cell-derived collagenase stimulatory factor (renamed EMMPRIN) is a member of the immunoglobulin superfamily. Cancer Res 55: 434-439, 1995.

23. Schmidt R, Bültmann A, Ungerer M, Joghetaei N, Bülbül O, Thieme S, Chavakis T, Toole BP, Gawaz M, Schömig A and May AE: Extracellular matrix metalloproteinase inducer regulates matrix metalloproteinase activity in cardiovascular cells: Implications in acute myocardial infarction. Circulation 113: 834-841, 2006.

24. Yoon YW, Kwon HM, Hwang KC, Choi EY, Hong BK, Kim D, Kim HS, Cho SH, Song KS and Sangiorgi G: Upstream regulation of matrix metalloproteinase by EMMPRIN; Extracellular matrix metalloproteinase inducer in advanced atherosclerotic plaque. Atherosclerosis 180: 37-44, 2005.

25. Ghattas A, Griffiths HR, Devitt A, Lip GY and Shantsila E: Monocytes in coronary artery disease and atherosclerosis: Where are we now? J Am Coll Cardiol 62: 1541-1551, 2013.

26. Chen F, Eriksson P, Hansson GK, Herzfeld I, Klein M, Hansson LO and Valen G: Expression of matrix metalloproteinase 9 and its regulators in the unstable coronary atherosclerotic plaque. Int J Mol Med 15: 57-65, 2005.

27. Nakashima Y, Plump AS, Raines EW, Breslow JL and Ross R: ApoE-deficient mice develop lesions of all phases of atherosclerosis throughout the arterial tree. Arterioscler Thromb 14: 133-140, 1994.

28. Jawien J, Nastalek P and Korbut R: Mouse models of experimental atherosclerosis. J Physiol Pharmacol 55: 503-517, 2004.

29. Johnson J, Carson K, Williams H, Karanam S, Newby A, Angelini G, George S and Jackson C: Plaque rupture after short periods of fat feeding in the apolipoprotein E-knockout mouse: Model characterization and effects of pravastatin treatment. Circulation 111: 1422-1430, 2005.

30. Rosenfeld ME, Carson KG, Johnson JL, Williams H, Jackson CL and Schwartz SM: Animal models of spontaneous plaque rupture: The holy grail of experimental atherosclerosis research. Curr Atheroscler Rep 4: 238-242, 2002.

31. Gao P, Guo RW, Chen JF, Chen Y, Wang H, Yu Y and Huang L: A meprin inhibitor suppresses atherosclerotic plaque formation in $\mathrm{ApoE}^{-/}$mice. Atherosclerosis 207: 84-92, 2009.
32. Cheng XW, Kuzuya M, Sasaki T, Arakawa K, Kanda S, Sumi D, Koike T, Maeda K, Tamaya-Mori N, Shi GP, et al: Increased expression of elastolytic cysteine proteases, cathepsins $\mathrm{S}$ and $\mathrm{K}$, in the neointima of balloon-injured rat carotid arteries. Am J Pathol 164: 243-251, 2004.

33. Grainger DJ, Witchell CM and Metcalfe JC: Tamoxifen elevates transforming growth factor-beta and suppresses diet-induced formation of lipid lesions in mouse aorta. Nat Med 1: 1067-1073, 1995.

34. Sukhova GK, Zhang Y, Pan JH, Wada Y, Yamamoto T, Naito M, Kodama T, Tsimikas S, Witztum JL, Lu ML, et al: Deficiency of cathepsin $\mathrm{S}$ reduces atherosclerosis in LDL receptor-deficient mice. J Clin Invest 111: 897-906, 2003

35. Zhang J, Ge H, Wang C, Guo TB, He Q, Shao Q and Fan Y: Inhibitory effect of PPAR on the expression of EMMPRIN in macrophages and foam cells. Int J Cardiol 117: 373-380, 2007.

36. Seizer P, Schonberger T, Schött M, Lang MR, Langer HF, Bigalke B, Krämer BF, Borst O, Daub K, Heidenreich O, et al: EMMPRIN and its ligand cyclophilin A regulate MT1-MMP, MMP-9 and M-CSF during foam cell formation. Atherosclerosis 209: 51-57, 2010.

37. Gabison EE, Hoang-Xuan T, Mauviel A and Menashi S: EMMPRIN/CD147, an MMP modulator in cancer, development and tissue repair. Biochimie 87: 361-368, 2005.

38. Haug C, Lenz C, Díaz F and Bachem MG: Oxidized low-density lipoproteins stimulate extracellular matrix metalloproteinase Inducer (EMMPRIN) release by coronary smooth muscle cells. Arterioscler Thromb Vasc Biol 24: 1823-1829, 2004.

39. Dutta P, Courties G, Wei Y, Leuschner F, Gorbatov R, Robbins CS, Iwamoto Y, Thompson B, Carlson AL, Heidt T, et al: Myocardial infarction accelerates atherosclerosis. Nature 487: 325-329, 2012.

40. Kampoli AM, Tousoulis D, Papageorgiou N, Antoniades C, Androulakis E, Tsiamis E, Latsios G and Stefanadis C: Matrix metalloproteinases in acute coronary syndromes: Current perspectives. Curr Top Med Chem 12: 1192-1205, 2012.

41. Satoh K, Nigro P, Matoba T, O'Dell MR, Cui Z, Shi X, Mohan A, Yan C, Abe J, Illig KA and Berk BC: Cyclophilin A enhances vascular oxidative stress and the development of angiotensin II-induced aortic aneurysms. Nat Med 15: 649-656, 2009.

42. Sukhanov S, Higashi Y, Shai SY, Vaughn C, Mohler J, Li Y, Song YH, Titterington J and Delafontaine P: IGF-1 reduces inflammatory responses, suppresses oxidative stress, and decreases atherosclerosis progression in ApoE-deficient mice. Arterioscler Thromb Vasc Biol 27: 2684-2690, 2007.

43. Newby AC: Metalloproteinases and vulnerable atherosclerotic plaques. Trends Cardiovasc Med 17: 253-258, 2007.

44. Nie R, Xie S, Du B, Liu X, Deng B and Wang J: Extracellular matrix metalloproteinase inducer (EMMPRIN) is increased in human left ventricle after acute myocardial infarction. Arch Med Res 40: 605-611, 2009.

45. Fiotti N, Altamura N, Orlando C, Simi L, Reimers B, Pascotto P, Zingone B, Pascotto A, Serio M, Guarnieri G and Giansante C: Metalloproteinases-2, -9 and TIMP-1 expression in stable and unstable coronary plaques undergoing PCI. Int J Cardiol 127: 350-357, 2008.

46. Yang LX, Yang ZH, Guo RW, Ye JS and Liu H: Angiotensin II induces extracellular matrix metalloproteinase inducer expression via an AT1R dependent pathway in aortic atherosclerotic plaque in apolipoprotein E knockout mice. J Renin Angiotensin Aldosterone Syst 13: 67-75, 2012.

47. Bea F, Blessing E, Bennett B, Levitz M, Wallace EP and Rosenfeld ME: Simvastatin promotes atherosclerotic plaque stability in apoE-deficient mice independently of lipid lowering. Arterioscler Thromb Vasc Biol 22: 1832-1837, 2002.

48. Ray KK and Cannon CP: Atorvastatin and cardiovascular protection: A review and comparison of recent clinical trials. Expert Opin Pharmacother 6: 915-927, 2005.

49. Shao Q, Shen LH, Hu LH, Pu J, Jing Q and He B: Atorvastatin suppresses inflammatory response induced by oxLDL through inhibition of ERK phosphorylation, I $\kappa \mathrm{B} \alpha$ degradation, and $\mathrm{COX}-2$ expression in murine macrophages. J Cell Biochem 113: 611-618, 2012.

50. Deng P, Zhao SP, Dai HY, Guan XS and Huang HG: Atorvastatin reduces the expression of COX-2 mRNA in peripheral blood monocytes from patients with acute myocardial infarction and modulates the early inflammatory response. Clin Chem 52: 300-303, 2006. 